\title{
Taking six-dimensional spectra in finite time
}

\section{Citation}

Goodknight, Joseph, and Alan Aspuru-Guzik. 2017. “Taking Six-Dimensional Spectra in Finite Time." Science 356 (6345) (June 29): 1333-1333. doi:10.1126/science.aan2842.

\section{Published Version}

10.1126/science.aan2842

\section{Permanent link}

http://nrs.harvard.edu/urn-3:HUL.InstRepos:33439170

\section{Terms of Use}

This article was downloaded from Harvard University's DASH repository, and is made available under the terms and conditions applicable to Other Posted Material, as set forth at http:// nrs.harvard.edu/urn-3:HUL.InstRepos:dash.current.terms-of-use\#LAA

\section{Share Your Story}

The Harvard community has made this article openly available.

Please share how this access benefits you. Submit a story.

Accessibility 


\title{
Taking six-dimensional spectra in finite time
}

\author{
Clever data acquisition can probe how vibrations couple to electronic states in \\ molecules \\ By Joseph Goodknight and Alan Aspuru-Guzik
}

Pump-probe spectroscopies use ultrafast laser pulses to excite molecules, and then probe them with a time-delayed pulse. If more laser pulses are used, information beyond excited-state spectra can be gained. For example, four-pulse techniques can explore how vibra-tions or electronic excited states are coupled. However, adding even more pulses usually comes at a price, that of very long experi-mental times to collect all of the data. Spencer et al. (1) now report on a six-pulse laser spec-troscopy that obtains intricate details on the coupling between electronic states to vibra-tional motions, a key issue in the observation of quantum coherence in proteins (2-5). This method takes advantage for the ingenious gradient-assisted photon-echo spectroscopy (GRAPES) developed (6) for four-pulse spectros-copy that speeds acquisition times by tilting the beams to introduce time delays and sam-pling along a line rather than a point.

In so-called two-dimensional (2D) spec-troscopy, two time dimensions are scanned, one in between the two pumps and the other between the two probes. A "map" is generat-ed of the energy flow in the system as a function of time. As laser pulses become fast-er, the energy range they must cover neces-sarily increases because of the Uncertainty Principle. Thus, probing a typical vibration transition requires a pulse about 100 femto-seconds (10-15 s or fs) long, but an electronic transition of a molecule requires a pulse clos-er to $50 \mathrm{fs}$ long. Thus, 2D optical spectroscopy first addressed the coupling of chemical vi-brations (7), and as faster pulses were devel-oped, could probe electronic energy transfer in many different systems, including photo-synthetic light harvesting systems (2-4).

The experiments on photosynthetic com-plexes suggested that in certain cases, elec-tronic excited states might be in a quantum coherent superposition that lasted long enough to transport energy like a wave, in-stead of like a particle-which would be slow-er-through an array of chromophores. If true, biomolecular engineering for sustaining wavelike transport would be a of interest for basic and applied research. One use could be better light collection devices. Other studies, however, have emphasized the role of mo-lecular vibrations to explain these observa-tions (5) To help in settling these long-standing discussions, new spectroscop-ic techniques that allow for extracting more information need to be developed.

The GAMERS method (gradient-assisted multidimensional electronic Raman spec-troscopy) may provide a way to settle this matter by adding another pulse to a 2D elec-tronic spectroscopy. This additional pulse controls the initial vibrational state of the molecule. During the time between the first and the second pulse, the vibrational state changes. By looking at the 
subsequent chang-es to the electronic coupling based on the starting vibrational state, GAMERS could de-termine how vibrations change the electronic coupling, which is not generally directly ob-tained from other spectroscopies.

With a traditional setup, a full set of data collection for a GAMERS experiment would require more than 1 week to collect all nec-essary data points. That is technically very challenging because state-of-the-art lasers are not guaranteed to be stable enough for that long. Instead of delaying two beams by physically changing the beam path and measuring in the same location, GRAPES tilts the two beams relative to each other (see the figure) and takes the detection as a 2D image, thus performing all of the necessary experiments at once and drastically reducing the time to solution. GAMERS uses the GRAPES technique for the delay between the middle two pulses, thus reducing the acquisi-tion time from 1 week to mere hours. The complex multidimensional spectroscopic sig-nals acquired may obtain intricate details on the coupling of electronic states to nuclear vibrational motion. The quantum of perov-skite solar cells (8), singlet-fission materials (9), quantum nanostructures (10), and photo-synthestic complexes (2) all exhibit details of the electronic-vibrational interactions that require further exploration. There are some drawbacks, however. The GAMERS protocol requires a molecular vi-bration that is Raman-active. A Raman ac-tive vibration is one that allows light to scat-ter, losing energy to the vibration, but not every vibration can accomplish this with a sufficiently strong signal. There is also theo-retical work needed to understand precisely what information GAMERS encodes. It may be particularly useful to consider GAMERS in the context of quantum-process tomography (11), as the addition of more pulses makes the experiment probe more elements of the quantum process matrix.

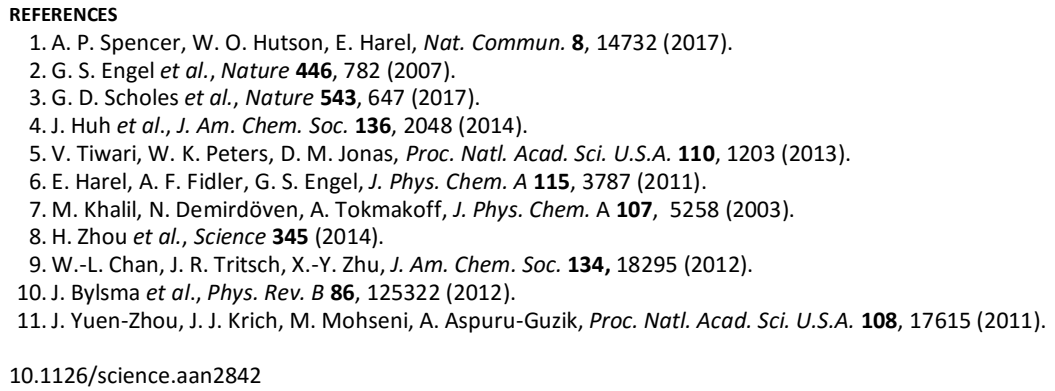

Figure 1: Reprinted and adapted from the original paper (1). a The top is a diagram of the 5 separate laser pulses focusing in to the target. The bottom part of the figure showing pulse the pulses coming in to the system; it also gives a sense for the experimental complexity involved: at minimum, T0, and T all have to be scanned without GRAPES (11). Note the tilt between beams 1 and 2 in the circular inset, which is GRAPES' hallmark. Also note that the 0th pulse interacts with the system twice, which is why GAMERS is considered a 6-pulse experiment. B An example of the kind of 4-dimensional data they get, showing correlations between energy levels wherever there is signal where the pump $(x)$ frequency is different from the probe(y) frequency. 
a

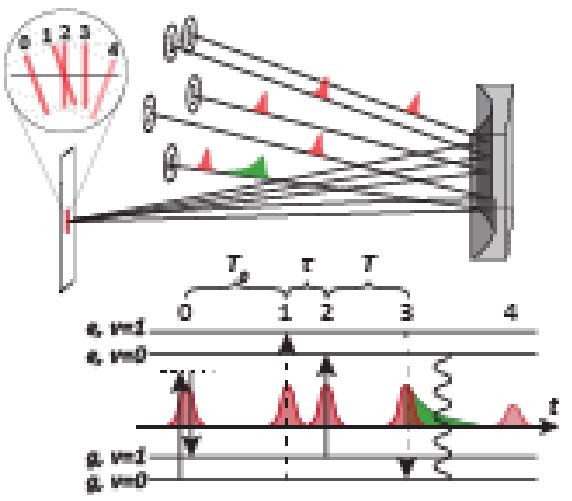

b

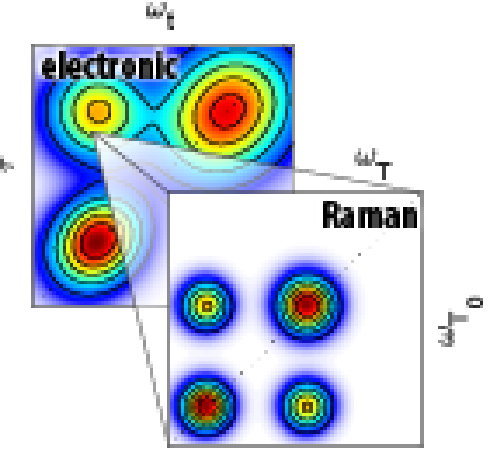

